\title{
Vegetation cover-wind erosion correlation: A case study
}

\author{
Mücahit Karaoğlu ${ }^{1 *}$, Erhan Erdel ${ }^{1}$ \\ ${ }^{1 *}$ Iğdır University, Agricultural Faculty, Department of Soil Science and Plant Nutrition, Iğdır, Turkey, (ORCID: 0000-0002-7388-9192), mucahit.karaoglu@igdir.edu.tr \\ ${ }^{1}$ Iğdır University, Agricultural Faculty, Department of Soil Science and Plant Nutrition, Iğdır, Turkey, (ORCID: 0000-0002-8011-9452), erhan.erdel@igdir.edu.tr
}

(First received 21 June 2021 and in final form 13 November 2021)

(DOI: $10.31590 /$ ejosat.955460)

ATIF/REFERENCE: Çelik, A., Akça, E. 2021. Description of the quarter-century effect of conversion from rainfed Farming to irrigated farming on a micromorphological scale. European Journal of Science and Technology, (27), 925-934.

\begin{abstract}
Plants, indispensable in all respects for other living things, are also indispensable for the inanimate soil on which they grow and feed. A land devoid of vegetation is vulnerable both in terms of itself and the many animal species living there. In this study the relationship between vegetation cover and wind erosion processes was investigated. A total of 57 soil samples were taken in different ways from areas protected and unprotected from wind erosion in Igdir-Aralik, which is the second largest wind erosion area in Turkey. For physical and chemical analyses of the samples, texture, carbonate, $\mathrm{pH}$, aggregate stability, organic matter, and electrical conductivity values were determined after dry sieving with $2 \mathrm{~mm}, 1 \mathrm{~mm}, 0.84 \mathrm{~mm}, 0.42 \mathrm{~mm}, 0.106 \mathrm{~mm}, 0.020 \mathrm{~mm}$ and $<0.020 \mathrm{~mm}$ sieve values. According to Duncan multiple comparison test results, most of the data were found to be statistically insignificant. These results revealed that the percentage of vegetation cover and conservation practices in study area are insufficient.
\end{abstract}

Key words: Vegetation cover and percentage, wind erosion, erodible aggregates, Iğdır-Aralik.

\section{Bitki örtüsü-rüzgâr erozyonu ilişkileri: Bir durum çalışması}

\section{$\ddot{O z}$}

Diğer canlılar için her bakımdan vazgeçilmez olan bitkiler, üzerinde büyüyüp beslendiği cansız toprak için de vazgeçilmez bir canlı türüdür. Bitki örtüsünden yoksun bir toprak hem kendisi hem de içinde barındırdığı pek çok hayvan türleri açısından savunmasız durumdadır. Bitki örtüsü ile rüzgâr erozyon süreçleri arasındaki ilişkinin araştırıldığı bu çalışmada Iğdır-Aralık’ta yer alan, Türkiye'nin ikinci büyük rüzgâr erozyon sahasının koruma çalışmaları yapılmış ve korumasız bölümlerinden farklı şekillerde 57 toprak örneği alınmıştır. Alınan örnekler üzerinde yürütülen fiziksel ve kimyasal analizlerde tekstür, kireç, pH, agregat stabilitesi, organik madde ve elektriksel iletkenlik değerleri; kuru elemelerde $2 \mathrm{~mm}, 1 \mathrm{~mm}, 0.84 \mathrm{~mm}, 0.42 \mathrm{~mm}, 0.106 \mathrm{~mm}, 0.020 \mathrm{~mm}$ ve $<0.020 \mathrm{~mm}$ değerleri belirlenmiştir. Duncan çoklu karşılaştırma testi sonuçlarına göre elde edilen verilerin büyük bir çoğunluğu istatistiksel olarak önemsiz bulunmuştur. Bu sonuçlar çalışma alanında bitki örtü yüzdesinin ve koruma önlemlerinin yetersiz olduğunu ortaya koymuştur.

Anahtar kelimeler: Bitki örtüsü ve yüzdesi, rüzgâr erozyonu, aşınabilir agregatlar, Iğdır-Aralık.

\footnotetext{
* Corresponding Author: mucahit.karaoglu@igdir.edu.tr
} 


\section{Introduction}

Seeing the soil as bare and inefficient and polluting it irresponsibly is a source of great shame for humanity as the soil serves as a home for many plant and animal species and directly and indirectly provides for many human needs. Despite the geometric increase in the world's population, the soil amount, especially fertile soil, remains the same and even decreases due to many reasons, especially accelerated erosion.

Soil fertility is related to soil quality. Soil quality is expressed as the physical, chemical, and biological properties of the soil. An important feature that should be known to evaluate soil quality and ensure plant growth is soil fertility [1]. Plants have direct and indirect effects on improving soil fertility and, as a result, soil quality and soil change processes. [2].

Soil has a continuous dynamic structure; that is physical and chemical events do not end. The multiplier effect of climate events on soil mobility directs those who deal with agriculture and soil to examine vegetation because the existence and density of vegetation is a reality that cannot be ignored for rich soil development in every aspect. In addition, one of the important tasks of some vegetation species is to meet the need for animal feed $[3,4]$.

In many countries around the world and especially in Turkey, insufficient vegetation is a serious agricultural and environmental problem. However, a bigger problem than this is negative behaviour such as anthropogenic forest fires, excessive and early grazing, lack of awareness and insensitivity to erosion. All these negative aspects cause a decrease in the existing vegetation cover and especially species and their density and therefore the amount of feed [5] in pastures managed without awareness. Consequently, the economic value of these plants decreases.

The most important cause of erosion, or more precisely accelerated erosion, is the devastation or destruction of vegetation which is indispensable for the soil and protects the soil and other living things in the soil like an umbrella. It was reported [6] that 432.2 million hectares of land in the world are threatened by wind erosion and these areas vary according to different factors (continents, climatic conditions, etc.). Wind erosion is particularly effective and dangerous in regions with arid and semi-arid climates. Wind erosion can also have negative effects on large areas like water erosion depending on the strength and continuity of the wind causing more soil material to be displaced. Worldwide the transport of soil particles by wind is also recognized as a serious environmental [7] and health problem.

It was stated that wind erosion is observed in approximately 500,000 hectares of land, ranging from mild to very severe in our country, and approximately $70 \%$ of this area is within the borders of Konya province [8]. In this study, which was carried out to investigate how vegetation affects wind erosion processes in Iğdır-Aralik, which is known as the second largest wind erosion area in Turkey, the analysis of soil samples and dry sieving results taken from different vegetation conditions, including where protective measures were applied but are currently abandoned, and control samples taken from bare soils were statistically compared and interpreted.

\section{Material and Method}

\subsection{Materials}

\subsubsection{Soil samples}

Disturbed soil samples used in the study were taken from surface soils were in protected and unprotected parts (located between $394752.4 ; 394720.9 ; 394950.1 ; 394906.7$ northern latitudes and 443529.9; 443415.8; 443222.2; 443119.8 eastern longitudes) of Igdir-Aralik wind erosion area [9]. Igdir-Aralik is Turkey's second largest wind erosion area and covers 13,554 hectares [10] to a depth of $0-5 \mathrm{~cm}$ [11], which is the most important level in terms of wind erosion. The proportion of aggregates in the uppermost part of the soil $(2.54 \mathrm{~cm})$ is defined as wind-erodible particles (EF) $[12,13]$, and therefore surface soil properties are emphasized in studies about wind erosion.

\subsubsection{Vibratory dry sieving device}

A Retsch AS 200 basic brand vibrating dry sieving device was used to determine the wind erosion sensitivity of surface soil samples and particle size distribution and to obtain soil particle fractions with different sizes as percentages. Sieves of $2 \mathrm{~mm}, 1$ $\mathrm{mm}, 0.840 \mathrm{~mm}, 0.420 \mathrm{~mm}, 0.106 \mathrm{~mm}$, and $0.020 \mathrm{~mm}$ diameter sieves were used [14]. Dry sieving is a standard method for determining some parameters related to soil aggregation and susceptibility to wind erosion. [15]. The size and durability of soil aggregates is the most important factor affecting soil sensitivity to wind erosion [16].

\subsection{Method}

\subsubsection{Soil sampling}

In the Igdir-Aralık wind erosion area, three different methods were used to sample soil and investigate whether the existing but insufficient vegetation has an effect or not on erosion caused by strong winds between March and October:

Case 1: Acacia (Acacia albida) trees $2.5-3 \mathrm{~m}$ tall were planted at $25 \mathrm{~m}$ intervals [17] in double row strips and the space between the strips were $1.5 \mathrm{~m}$. Three samples and 3 repetitions at $75 \mathrm{~m}$ intervals from the middle of the double row strips; 3 samples and 3 repetitions at intervals of $75 \mathrm{~m}$ from the inside of the strips; randomly, and 3 control samples from bare soils (Figure 1) for comparison were collected for a total of 21 (7x3) samples. Considering that preservation would be higher while sampling inside the strips, sampling began from the 2nd strip, from the middle of the 1st and 2nd strips while sampling between the double row strips. In other words, the samples were taken from northwest to southeast and the first samples from southwest to northeast. 


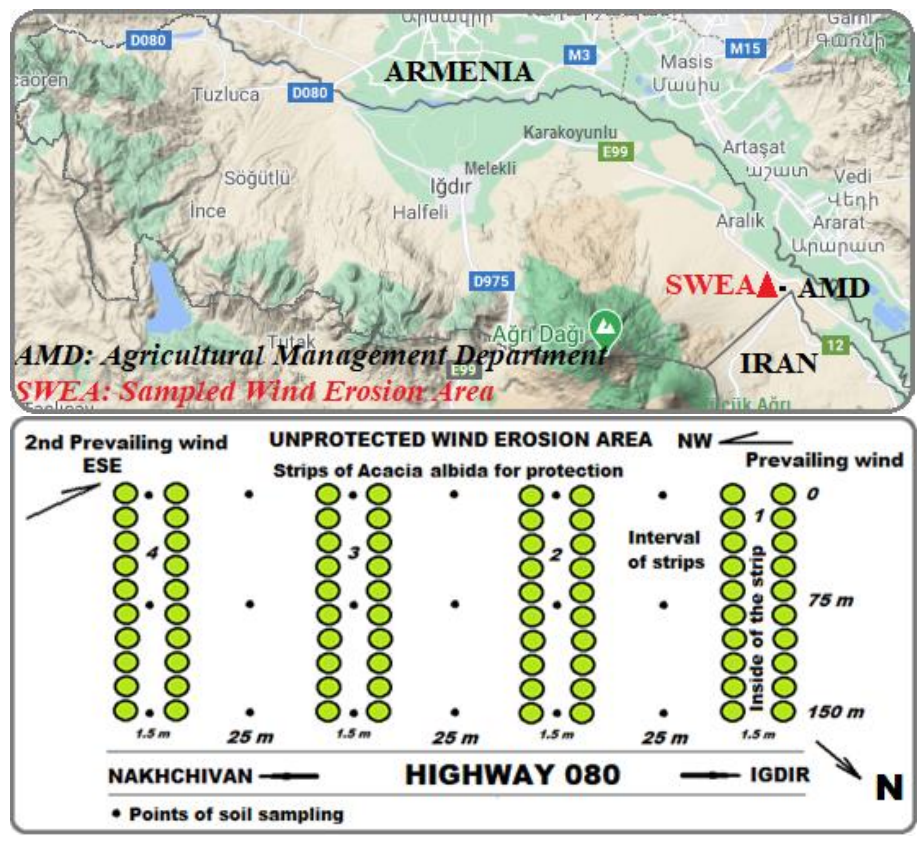

Figure 1. Sampling area and sampling plan for Case 1

Case 2: Ebu cehil (Ephedra distachya L.) bush, the most common natural plant species in the Iğdır-Aralık wind erosion area, is a plant species from the Ephedraceae family ranging in height from 25 to $50 \mathrm{~cm}$ which protects the soil against erosion with strong roots and is known as a strong stimulant. For this plant, samples from near plants with crown widths of 100, 150 and $250 \mathrm{~cm}$ ones were taken from windward, bush bottom, and leeward (Figure 2) sides. There were 3 samples and 3 repetitions with 3 control samples from bare soils for comparison for a total of 30 (10x3) samples.

Case 3: To investigate whether the Ebu cehil bushes, which developed between the acacia strips with crown width $230 \mathrm{~cm}$, provide more protection or not, a total of 12 samples $(4 \times 3)$ were collected as in case 2 (Figure 2) from windward, bush bottom, leeward points with 3 samples and 3 repetitions and 3 control samples from bare soils for comparison.

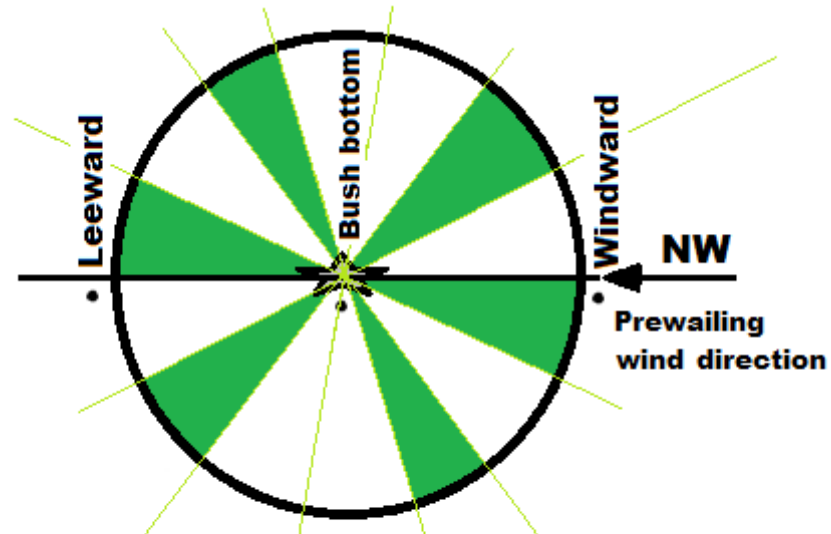

CROWN WIDTH

- Points of soil sampling

Figure 2. Sampling plan for Case 2 and 3

\subsubsection{Physical and chemical analysis of soil samples}

A proportion of the disturbed soil samples taken from the wind erosion area were sieved to $2 \mathrm{~mm}$ in a dry air environment. Texture (\%) was determined by the hydrometer method [18]. Soil reaction $(\mathrm{pH})$ was measured potentiometrically in 1:2.5 soil-water suspension with a "glass electrode" pH meter [19]. Lime (\%) was measured volumetrically with a Scheibler Calcimeter [20]. Organic matter (\%) was measured with the Walkley-Black method [21] and electrical conductivity (EC.103) was examined by obtaining extraction solutions from the prepared saturation pastes with an electrical conductivity instrument in $\mathrm{dS} \mathrm{cm}^{-1}$ [22].

\subsubsection{Dry sieving processes}

It is a well-known and accepted finding that soil aggregates larger than $0.84 \mathrm{~mm}$ in diameter are resistant to wind erosion [23]. The most important wind erosion process is saltation by particles larger than the aggregate diameter of $0.106 \mathrm{~mm}$. The immobile surfaces of clods and crusts are eroded after saltation begins and if saltation continues, new saltation, drifting and suspended $(<0.106 \mathrm{~mm})$ particles are created through kinetic energy transfer [24].

The dry sieving process was applied to the soil samples of 200 grams at $50 \mathrm{~Hz}$ vibration frequency for 5 minutes and the soil material sieved through $2 \mathrm{~mm}, 1 \mathrm{~mm}, 0.840 \mathrm{~mm}, 0.420 \mathrm{~mm}, 0.106$ $\mathrm{mm}$, and $0.020 \mathrm{~mm}$ diameter sieves was determined as percentages for each soil sample.

\subsubsection{Determination of erodible material}

The wind-erodible particles at the top of the soil $(0-2.54$ $\mathrm{cm})$ are defined as the erodible fraction $(\mathrm{EF})$. EF is the percentage of particles smaller than $0.84 \mathrm{~mm}$ and are considered sensitive soil particles that can be transported by wind [25]. EF is used as the potential abrasion index in the wind erosion equation (WEQ) and the revised wind erosion equation (RWEQ). The EF value varies as a function of soil texture [26], organic matter content [27], and free $\mathrm{CaCO}_{3}$ content [28]. To determine the amount of material that can be eroded as a percentage two different equations were used.

[13] and

$$
E F_{1}=\left(W_{<0.84} / T W\right) \times 100
$$

$$
\begin{gathered}
E F_{2}=29.9+0.31 \text { Sand }+0.17 \text { Silt }+ \\
0.33 \text { Sand/Clay }-2.590 \mathrm{M}-0.95 \mathrm{CaCO}_{3}
\end{gathered}
$$

[12] and the erosion susceptibility of the samples were investigated. Here, EF is the amount of erodible fraction (g), $\mathrm{W}_{<0,84}$ is the amount of material less than $0.84 \mathrm{~mm}(\mathrm{~g}), \mathrm{TW}$ is the total weight of soil sample, OM is organic matter content (\%) and $\mathrm{CaCO}_{3}$ is lime content $(\%)$.

\subsubsection{Statistical analysis}

In vegetation-wind erosion correlations, the Duncan multiple comparison test was applied to the obtained data in order 
to reveal the significance level of the changes in the results, especially the significance level of possible increase in resistant aggregation $(>0.84 \mathrm{~mm}$ ), the significance level of the change in the amount of erodible material $(\mathrm{EF})$, of particles $(0.106 \mathrm{~mm})$ that trigger erosion by saltation and the physical and chemical properties of soil samples.

In this method developed by Duncan in 1951, the average of each procedure is compared with all other averages and individual test values. During the application of the Duncan test, the $\mathrm{F}$ values calculated by variance analysis of the procedures do not have to be statistically significant. Therefore, this method can be applied in both cases without any problems. This is an advantage of this test [29].

\section{Results and Discussion}

\subsection{Analysis and Dry Sieving Results}

The results of triplicate physical and chemical analysis of all soil samples used in the study are given in Tables 1, 3 and 5 for cases 1, 2 and 3, respectively. The dry sieving results for cases 1 , 2 and 3 are shown in Tables 2, 4 and 6, respectively.

Table 1. Analysis results (Case 1)

\begin{tabular}{|c|c|c|c|c|c|c|c|c|c|c|c|}
\hline \multicolumn{2}{|c|}{ Samples } & Clay* & Silt* & Sand* & T. C. & Lime* & pH & E.C. $10^{3}$ & A. S.* & O. M.* & $\mathbf{E F}_{2}{ }^{*}$ \\
\hline \multirow{3}{*}{ Control } & 1 & 6 & 11 & 83 & LS & 0.24 & 7.74 & 220.34 & 15.8 & 0.27 & 61.19 \\
\hline & 2 & 6 & 12 & 82 & LS & 0.98 & 7.71 & 75.56 & 11.3 & 0.8 & 58.88 \\
\hline & 3 & 2 & 10 & 88 & $\mathrm{~S}$ & 0.40 & 8.12 & 272.00 & 18.18 & 0.87 & 70.76 \\
\hline \multirow{3}{*}{$\begin{array}{c}\text { Acacia } \\
\text { BS-1 }\end{array}$} & 1 & 0 & 12 & 88 & $\mathrm{~S}$ & 0.80 & 8.36 & 280.00 & 11.82 & 0.92 & 64.99 \\
\hline & 2 & 2 & 34 & 64 & SL & 0.56 & 8.93 & 173.00 & 20.67 & 1.05 & 76.36 \\
\hline & 3 & 6 & 32 & 62 & SL & 0.24 & 8.10 & 208.00 & 16.23 & 1.04 & 60.09 \\
\hline \multirow{3}{*}{$\begin{array}{c}\text { Acacia } \\
\text { BS-2 }\end{array}$} & 1 & 2 & 8 & 90 & $\mathrm{~S}$ & 0.48 & 8.64 & 98.00 & 11.30 & 0.84 & 71.04 \\
\hline & 2 & 2 & 14 & 84 & S & 0.48 & 8.30 & 103.00 & 16.67 & 0.76 & 69.76 \\
\hline & 3 & 2 & 22 & 76 & LS & 0.32 & 8.05 & 226.00 & 11.79 & 0.89 & 67.13 \\
\hline \multirow{3}{*}{$\begin{array}{c}\text { Acacia } \\
\text { BS-3 }\end{array}$} & 1 & 4 & 18 & 78 & LS & 0.08 & 8.73 & 89.00 & 16.47 & 1.04 & 58.99 \\
\hline & 2 & 2 & 18 & 80 & LS & 0.40 & 8.87 & 120.00 & 10.61 & 1.01 & 67.96 \\
\hline & 3 & 4 & 20 & 76 & LS & 0.16 & 8.82 & 167.00 & 20.10 & 1.05 & 60.26 \\
\hline \multirow{3}{*}{$\begin{array}{c}\text { Acacia } \\
\text { IS-2 }\end{array}$} & 1 & 2 & 16 & 82 & LS & 0.08 & 8.51 & 94.00 & 14.02 & 0.91 & 69.14 \\
\hline & 2 & 2 & 22 & 76 & LS & 0.16 & 8.12 & 84.00 & 21.39 & 0.84 & 66.65 \\
\hline & 3 & 2 & 22 & 76 & LS & 0.24 & 8.12 & 90.00 & 10.19 & 0.82 & 67.39 \\
\hline \multirow{3}{*}{$\begin{array}{c}\text { Acacia } \\
\text { IS-3 }\end{array}$} & 1 & 4 & 16 & 80 & $\mathrm{LS}$ & 0.16 & 8.52 & 84.00 & 14.77 & 0.92 & 61.48 \\
\hline & 2 & 2 & 16 & 82 & LS & 0.00 & 8.22 & 103.00 & 7.23 & 0.82 & 69.45 \\
\hline & 3 & 2 & 14 & 84 & LS & 0.08 & 7.96 & 103.00 & 12.11 & 0.86 & 69.88 \\
\hline \multirow{3}{*}{$\begin{array}{c}\text { Acacia } \\
\text { IS-4 }\end{array}$} & 1 & 3 & 24 & 73 & SL & 0.32 & 8.61 & 95.00 & 9.38 & 0.86 & 62.00 \\
\hline & 2 & 2 & 18 & 80 & LS & 0.16 & 8.20 & 76.00 & 17.89 & 1.02 & 68.17 \\
\hline & 3 & 2 & 22 & 76 & LS & 0.72 & 7.82 & 88.00 & 11.54 & 0.86 & 66.83 \\
\hline
\end{tabular}

*Values are given as percentage. T.C.: Texture class; E.C.10 ${ }^{3}$ Electrical conductivity; A.S.: Aggregate stability;

O.M.: Organic matter; $\mathrm{FF}_{2}$ : Erodible fraction; BS: Between the stripes; IS: Inside of the stripes.

Table 2. Dry Sieving results (Case 1)

\begin{tabular}{cccccccccc}
\hline Samples & & $\mathbf{2} \mathbf{~ m m}$ & $\mathbf{1 ~} \mathbf{~ m m}$ & $\mathbf{0 . 8 4} \mathbf{~ m m}$ & $\mathbf{0 . 4 2} \mathbf{~ m m}$ & $\mathbf{0 . 1 0 6} \mathbf{~ m m}$ & $\mathbf{2 0} \boldsymbol{\mu}$ & $<\mathbf{0 . 0 2 0} \boldsymbol{\mu}$ & $\mathbf{E F}_{\mathbf{1}}$ \\
\hline \multirow{2}{*}{ Control } & $\mathbf{1}$ & 0.06 & 1.07 & 0.65 & 9.15 & 71.93 & 15.80 & 1.34 & 98.22 \\
& $\mathbf{2}$ & 0.01 & 0.19 & 0.28 & 13.74 & 73.51 & 11.87 & 0.40 & 99.52 \\
& $\mathbf{3}$ & 0.44 & 2.44 & 0.33 & 17.44 & 54.53 & 21.52 & 3.30 & 96.79 \\
\hline \multirow{2}{*}{ Acacia } & $\mathbf{1}$ & 0.00 & 1.14 & 0.79 & 20.13 & 56.81 & 20.10 & 1.04 & 98.08 \\
BS-1 & $\mathbf{2}$ & 2.87 & 8.15 & 1.32 & 13.22 & 45.12 & 25.92 & 3.39 & 87.65 \\
& $\mathbf{3}$ & 1.36 & 6.00 & 0.55 & 16.58 & 46.77 & 25.06 & 3.68 & 92.08 \\
\hline \multirow{2}{*}{ Acacia } & $\mathbf{1}$ & 0.54 & 3.91 & 0.32 & 11.25 & 58.19 & 24.30 & 1.48 & 95.22 \\
BS-2 & $\mathbf{2}$ & 1.26 & 3.06 & 0.46 & 17.32 & 58.89 & 17.50 & 1.51 & 95.22 \\
& $\mathbf{3}$ & 2.45 & 7.83 & 0.35 & 12.87 & 45.61 & 26.62 & 4.27 & 89.37 \\
\hline \multirow{2}{*}{ Acacia } & $\mathbf{1}$ & 0.24 & 0.87 & 0.18 & 19.30 & 66.71 & 12.01 & 0.68 & 98.71 \\
BS-3 & $\mathbf{2}$ & 1.84 & 7.52 & 0.47 & 24.64 & 50.66 & 13.34 & 1.53 & 90.17 \\
& $\mathbf{3}$ & 3.95 & 8.12 & 0.29 & 14.47 & 50.89 & 19.07 & 3.20 & 87.63 \\
\hline \multirow{2}{*}{ Acacia } & $\mathbf{1}$ & 0.10 & 0.22 & 0.09 & 8.97 & 72.84 & 17.07 & 0.71 & 99.59 \\
IS-2 & $\mathbf{2}$ & 3.76 & 7.62 & 0.70 & 19.00 & 46.01 & 20.89 & 2.01 & 87.91 \\
& $\mathbf{3}$ & 2.52 & 7.30 & 0.49 & 20.80 & 47.36 & 18.68 & 2.86 & 89.70 \\
\hline \multirow{2}{*}{ Acacia } & $\mathbf{1}$ & 3.41 & 4.80 & 0.27 & 13.64 & 53.92 & 21.20 & 2.76 & 91.52 \\
IS-3 & $\mathbf{2}$ & 1.90 & 6.74 & 0.45 & 18.32 & 47.18 & 21.48 & 3.93 & 90.91 \\
& $\mathbf{3}$ & 2.40 & 5.49 & 0.38 & 15.51 & 51.63 & 20.41 & 4.19 & 91.74 \\
\hline \multirow{2}{*}{ Acacia } & $\mathbf{I}$ & 0.51 & 2.30 & 0.27 & 13.87 & 57.15 & 22.43 & 3.47 & 96.92 \\
IS-4 & $\mathbf{2}$ & 3.24 & 7.58 & 0.38 & 18.22 & 45.06 & 21.83 & 3.70 & 88.81 \\
& $\mathbf{3}$ & 2.53 & 9.38 & 0.37 & 21.85 & 43.72 & 19.32 & 2.84 & 87.73 \\
\hline
\end{tabular}

EF $F_{1}$ : Erodible fraction; BS: Between the strips; IS: Inside the strips. 
European Journal of Science and Technology

Table 3. Analysis results (Case 2)

\begin{tabular}{|c|c|c|c|c|c|c|c|c|c|c|c|}
\hline Sampl & & Clay* & Silt* & Sand* & T. C.* & Lime* & pH & E.C. $10^{3}$ & A. S.* & O. M.* & $\mathbf{E F}_{2}{ }^{*}$ \\
\hline \multirow{3}{*}{ Control } & 1 & 6 & 11 & 83 & $\mathrm{LS}$ & 0.24 & 7.74 & 220.34 & 15.8 & 0.27 & 61.19 \\
\hline & 2 & 6 & 12 & 82 & LS & 0.98 & 7.71 & 75.56 & 11.3 & 0.8 & 58.88 \\
\hline & 3 & 2 & 10 & 88 & $\mathrm{~S}$ & 0.40 & 8.12 & 272.00 & 18.18 & 0.87 & 70.76 \\
\hline \multirow{3}{*}{$\begin{array}{l}\text { E.D. } \\
100 \mathrm{~cm}(1)\end{array}$} & $W$ & 1 & 10 & 89 & $\mathrm{~S}$ & 0.08 & 7.67 & 94.00 & 21.43 & 1.04 & 85.79 \\
\hline & $B B$ & 3 & 24 & 73 & SL & 0.16 & 7.22 & 436.00 & 18.48 & 1.01 & 61.76 \\
\hline & $L$ & 3 & 22 & 75 & SL & 0.16 & 7.45 & 314.00 & 20.00 & 1.06 & 62.24 \\
\hline \multirow{3}{*}{$\begin{array}{l}\text { E.D. } \\
100 \mathrm{~cm}(2)\end{array}$} & $\boldsymbol{W}$ & 1 & 20 & 79 & LS & 0.16 & 7.41 & 206.00 & 7.41 & 0.85 & 81.51 \\
\hline & $\boldsymbol{B} B$ & 1 & 14 & 85 & LS & 0.08 & 7.60 & 63.00 & 7.73 & 0.74 & 84.69 \\
\hline & $L$ & 3 & 24 & 73 & SL & 0.24 & 7.66 & 105.00 & 22.40 & 1.05 & 61.58 \\
\hline \multirow{3}{*}{$\begin{array}{l}\text { E.D. } \\
100 \mathrm{~cm}(3)\end{array}$} & $\boldsymbol{W}$ & 3 & 16 & 81 & LS & 0.24 & 7.41 & 409.00 & 18.33 & 0.87 & 64.16 \\
\hline & $B B$ & 3 & 20 & 77 & LS & 0.08 & 7.59 & 86.00 & 15.44 & 0.92 & 63.29 \\
\hline & $L$ & 1 & 20 & 79 & LS & 0.16 & 7.71 & 87.00 & 14.41 & 0.91 & 81.35 \\
\hline \multirow{3}{*}{$\begin{array}{c}\text { E.D. } \\
150 \mathrm{~cm}(1)\end{array}$} & $W$ & 1 & 24 & 75 & $\mathrm{LS}$ & 0.16 & 7.43 & 250.00 & 9.01 & 0.72 & 79.96 \\
\hline & $\boldsymbol{B B}$ & 1 & 30 & 69 & SL & 0.16 & 7.79 & 111.00 & 12.41 & 0.86 & 76.78 \\
\hline & $L$ & 1 & 24 & 75 & LS & 0.24 & 7.76 & 86.00 & 18.95 & 0.85 & 79.55 \\
\hline \multirow{3}{*}{$\begin{array}{c}\text { E.D. } \\
150 \mathrm{~cm} \mathrm{(2)}\end{array}$} & $\boldsymbol{W}$ & 3 & 16 & 81 & $\mathrm{LS}$ & 0.24 & 7.42 & 430.00 & 8.21 & 0.84 & 64.24 \\
\hline & $B B$ & 1 & 26 & 73 & LS & 0.16 & 7.67 & 144.00 & 16.88 & 0.84 & 78.71 \\
\hline & $L$ & 3 & 20 & 77 & LS & 0.08 & 7.72 & 123.00 & 10.56 & 1.02 & 63.03 \\
\hline \multirow{3}{*}{$\begin{array}{c}\text { E.D. } \\
150 \mathrm{~cm} \mathrm{(3)}\end{array}$} & $\boldsymbol{W}$ & 3 & 16 & 81 & $\mathrm{LS}$ & 0.16 & 7.55 & 321.00 & 13.60 & 0.95 & 64.03 \\
\hline & $B B$ & 1 & 16 & 83 & LS & 0.08 & 7.77 & 103.00 & 12.72 & 0.86 & 83.44 \\
\hline & $L$ & 1 & 14 & 85 & LS & 0.16 & 7.83 & 95.00 & 11.50 & 0.84 & 84.35 \\
\hline \multirow{3}{*}{$\begin{array}{c}\text { E.D. } \\
250 \mathrm{~cm} \mathrm{(1)}\end{array}$} & $\boldsymbol{W}$ & 3 & 26 & 71 & SL & 0.24 & 7.71 & 236.00 & 18.00 & 0.96 & 61.54 \\
\hline & $B B$ & 1 & 16 & 83 & LS & 0.24 & 7.78 & 95.00 & 15.66 & 0.92 & 93.99 \\
\hline & $L$ & 1 & 14 & 85 & LS & 0.24 & 7.72 & 64.00 & 8.72 & 0.96 & 83.62 \\
\hline \multirow{3}{*}{$\begin{array}{c}\text { E.D. } \\
250 \mathrm{~cm}(2)\end{array}$} & $W$ & 1 & 22 & 77 & $\mathrm{LS}$ & 0.16 & 7.53 & 340.00 & 14.17 & 0.84 & 80.59 \\
\hline & $B B$ & 1 & 22 & 77 & LS & 0.32 & 7.64 & 95.00 & 11.59 & 0.85 & 80.41 \\
\hline & $L$ & 3 & 18 & 79 & LS & 0.24 & 7.80 & 94.00 & 13.39 & 0.82 & 63.68 \\
\hline \multirow{3}{*}{$\begin{array}{c}\text { E.D. } \\
250 \mathrm{~cm}(3)\end{array}$} & $\boldsymbol{W}$ & 1 & 16 & 83 & LS & 0.24 & 6.80 & 451.00 & 11.29 & 0.72 & 83.65 \\
\hline & $B B$ & 1 & 18 & 81 & LS & 0.24 & 7.81 & 92.00 & 14.50 & 0.64 & 83.38 \\
\hline & $L$ & 1 & 16 & 83 & LS & 0.32 & 7.53 & 99.00 & 9.42 & 0.75 & 83.49 \\
\hline
\end{tabular}

E.D.: Ephedra distachya L.; W: Windward; BB: Bush bottom; L: Leeward.

Table 4. Dry Sieving results (Case 2)

\begin{tabular}{|c|c|c|c|c|c|c|c|c|c|}
\hline \multicolumn{2}{|c|}{ Samples } & $2 \mathrm{~mm}$ & $1 \mathrm{~mm}$ & $0.84 \mathrm{~mm}$ & $0.42 \mathrm{~mm}$ & $0.106 \mathrm{~mm}$ & $20 \mu$ & $<0.020 \mu$ & $\mathbf{E F}_{1}$ \\
\hline \multirow{3}{*}{ Control } & 1 & 0.06 & 1.07 & 0.65 & 9.15 & 71.93 & 15.80 & 1.34 & 98.22 \\
\hline & 2 & 0.01 & 0.19 & 0.28 & 13.74 & 73.51 & 11.87 & 0.40 & 99.52 \\
\hline & 3 & 0.44 & 2.44 & 0.33 & 17.44 & 54.53 & 21.52 & 3.30 & 96.79 \\
\hline \multirow{3}{*}{$\begin{array}{l}\text { E.D. } \\
100 \mathrm{~cm} \mathrm{(1)}\end{array}$} & $\boldsymbol{W}$ & 2.17 & 8.50 & 0.70 & 24.28 & 52.43 & 9.92 & 2.00 & 88.63 \\
\hline & $B B$ & 3.55 & 7.27 & 0.47 & 19.23 & 57.96 & 10.94 & 0.59 & 88.72 \\
\hline & $L$ & 2.18 & 6.09 & 0.47 & 18.84 & 55.64 & 12.86 & 3.93 & 91.26 \\
\hline \multirow{3}{*}{$\begin{array}{c}\text { E.D. } \\
100 \mathrm{~cm}(2)\end{array}$} & $W$ & 2.31 & 6.87 & 0.64 & 12.69 & 62.36 & 10.89 & 4.25 & 90.19 \\
\hline & $B B$ & 1.21 & 3.64 & 0.45 & 17.78 & 62.37 & 12.04 & 2.52 & 94.70 \\
\hline & $L$ & 2.01 & 7.02 & 0.87 & 28.07 & 49.65 & 8.92 & 3.45 & 90.10 \\
\hline \multirow{3}{*}{$\begin{array}{l}\text { E.D. } \\
100 \mathrm{~cm}(3)\end{array}$} & $\boldsymbol{W}$ & 1.67 & 6.29 & 0.62 & 26.34 & 53.34 & 7.81 & 3.94 & 91.42 \\
\hline & $B B$ & 0.15 & 1.61 & 0.11 & 23.15 & 63.76 & 4.88 & 6.35 & 98.13 \\
\hline & $L$ & 1.89 & 8.03 & 0.67 & 26.50 & 51.74 & 8.03 & 3.14 & 89.41 \\
\hline \multirow{3}{*}{$\begin{array}{c}\text { E.D. } \\
150 \mathrm{~cm}(1)\end{array}$} & $W$ & 2.77 & 6.88 & 0.60 & 18.81 & 55.27 & 9.46 & 6.21 & 89.75 \\
\hline & $B B$ & 0.94 & 3.87 & 0.21 & 13.40 & 66.11 & 11.16 & 4.31 & 94.98 \\
\hline & $L$ & 3.99 & 7.69 & 0.55 & 20.38 & 51.83 & 9.14 & 6.42 & 87.77 \\
\hline \multirow{3}{*}{$\begin{array}{c}\text { E.D. } \\
150 \mathrm{~cm}(2)\end{array}$} & $\boldsymbol{W}$ & 2.30 & 5.26 & 0.19 & 18.58 & 57.85 & 15.83 & 0.00 & 92.26 \\
\hline & $B B$ & 0.40 & 1.60 & 0.15 & 12.60 & 70.53 & 11.49 & 3.22 & 97.85 \\
\hline & $L$ & 2.05 & 5.27 & 0.43 & 20.92 & 57.00 & 14.34 & 0.00 & 92.25 \\
\hline \multirow{3}{*}{$\begin{array}{c}\text { E.D. } \\
150 \mathrm{~cm}(3)\end{array}$} & $W$ & 3.25 & 8.25 & 0.42 & 23.49 & 52.29 & 12.29 & 0.00 & 88.08 \\
\hline & $B B$ & 0.50 & 1.52 & 0.12 & 15.63 & 71.52 & 10.71 & 0.00 & 97.87 \\
\hline & $L$ & 1.35 & 4.24 & 0.36 & 22.78 & 57.50 & 13.77 & 0.00 & 94.06 \\
\hline \multirow{3}{*}{$\begin{array}{c}\text { E.D. } \\
250 \mathrm{~cm}(1)\end{array}$} & $W$ & 2.73 & 7.67 & 0.40 & 21.00 & 52.49 & 15.71 & 0.00 & 89.19 \\
\hline & $B B$ & 1.29 & 6.40 & 0.61 & 27.09 & 54.53 & 10.08 & 0.00 & 91.70 \\
\hline & $L$ & 4.35 & 8.37 & 0.50 & 19.95 & 52.22 & 14.61 & 0.00 & 86.78 \\
\hline \multirow{3}{*}{$\begin{array}{c}\text { E.D. } \\
250 \mathrm{~cm}(2)\end{array}$} & $W$ & 5.05 & 8.17 & 0.37 & 18.30 & 53.11 & 14.99 & 0.00 & 86.40 \\
\hline & $B B$ & 1.54 & 4.15 & 0.22 & 13.96 & 62.35 & 17.78 & 0.00 & 94.09 \\
\hline & $L$ & 7.21 & 13.13 & 0.47 & 21.42 & 45.34 & 12.43 & 0.00 & 79.19 \\
\hline \multirow{3}{*}{$\begin{array}{c}\text { E.D. } \\
250 \mathrm{~cm}(3)\end{array}$} & $\boldsymbol{W}$ & 2.43 & 8.39 & 0.39 & 28.17 & 47.53 & 13.08 & 0.00 & 88.79 \\
\hline & $B B$ & 0.79 & 2.49 & 0.15 & 13.29 & 69.27 & 14.02 & 0.00 & 96.57 \\
\hline & $L$ & 3.94 & 8.62 & 0.35 & 24.36 & 49.08 & 13.65 & 0.00 & 87.09 \\
\hline
\end{tabular}

EF $F_{1}$ : Erodible fraction; E.D.: Ephedra distachya L.; W: Windward; BB: Bush bottom; L: Leeward. 
Table 5. Analysis results (Case 3)

\begin{tabular}{|c|c|c|c|c|c|c|c|c|c|c|c|}
\hline \multicolumn{2}{|c|}{ Samples } & Clay* & Silt* & Sand* & T. C. & Lime* & $\mathrm{pH}$ & E.C. $10^{3}$ & A. S.* & O. M.* & $\mathbf{E F}_{2}{ }^{*}$ \\
\hline \multirow{3}{*}{ Control } & 1 & 6 & 11 & 83 & $\mathrm{LS}$ & 0,24 & 7.74 & 220.34 & 15.8 & 0.27 & 61.19 \\
\hline & 2 & 6 & 12 & 82 & $\mathrm{LS}$ & 0.98 & 7.71 & 75.56 & 11.3 & 0.8 & 58.88 \\
\hline & 3 & 2 & 10 & 88 & $\mathrm{~S}$ & 0,40 & 8.12 & 272.00 & 18.18 & 0.87 & 70.76 \\
\hline B. Acacias & $W$ & 1 & 20 & 79 & LS & 0,08 & 7.86 & 122.00 & 5.56 & 0.74 & 81.87 \\
\hline E.D. & $\boldsymbol{B B}$ & 3 & 24 & 73 & SL & 0,32 & 8.13 & 288.00 & 19.91 & 0.98 & 61.69 \\
\hline $230 \mathrm{~cm}(1)$ & $L$ & 5 & 30 & 65 & $\mathrm{SL}$ & 0,16 & 7.91 & 89.00 & 11.16 & 1.01 & 56.67 \\
\hline B. Acacias & $\boldsymbol{W}$ & 3 & 24 & 73 & SL & 0,16 & 8.04 & 89.00 & 16.13 & 0.78 & 62.36 \\
\hline E.D. & $B B$ & 1 & 24 & 75 & LS & 0,16 & 8.01 & 480.00 & 22.65 & 1.08 & 79.03 \\
\hline $230 \mathrm{~cm}(2)$ & $L$ & 1 & 26 & 73 & SL & 0,24 & 7.73 & 163.00 & 7.72 & 0.81 & 78.71 \\
\hline B. Acacias & $\boldsymbol{W}$ & 3 & 14 & 83 & LS & 0,24 & 7.74 & 145.00 & 7.25 & 0.76 & 65.05 \\
\hline E.D. & $B B$ & 1 & 22 & 77 & LS & 0,08 & 7.55 & 259.00 & 10.62 & 0.81 & 80.75 \\
\hline $230 \mathrm{~cm}(3)$ & $L$ & 3 & 18 & 79 & LS & 0,16 & 7.85 & 194.00 & 11.73 & 0.72 & 64.01 \\
\hline
\end{tabular}

B: Between; E.D.: Ephedra distachya L.; W: Windward; BB: Bush bottom; L: Leeward.

Table 6. Dry Sieving results (Case 3)

\begin{tabular}{cccccccccc}
\hline \multicolumn{1}{c}{ Samples } & & $\mathbf{2 ~} \mathbf{~ m m}$ & $\mathbf{1} \mathbf{~} \mathbf{m}$ & $\mathbf{0 . 8 4} \mathbf{~ m m}$ & $\mathbf{0 . 4 2} \mathbf{~ m m}$ & $\mathbf{0 . 1 0 6} \mathbf{~ m m}$ & $\mathbf{2 0} \boldsymbol{\mu}$ & $<\mathbf{0 . 0 2 0} \boldsymbol{\mu}$ & $\mathbf{E F}_{\mathbf{1}}$ \\
\hline & $\boldsymbol{1}$ & 0.06 & 1.07 & 0.65 & 9.15 & 71.93 & 15.80 & 1.34 & 98.22 \\
Control & $\mathbf{2}$ & 0.01 & 0.19 & 0.28 & 13.74 & 73.51 & 11.87 & 0.40 & 99.52 \\
& $\mathbf{3}$ & 0.44 & 2.44 & 0.33 & 17.44 & 54.53 & 21.52 & 3.30 & 96.79 \\
\hline B. Acacias & $\boldsymbol{W}$ & 5.90 & 7.68 & 0.43 & 18.02 & 43.07 & 22.09 & 2.80 & 85.99 \\
E.D. & $\boldsymbol{B} \boldsymbol{B}$ & 3.49 & 9.91 & 0.28 & 19.47 & 38.39 & 23.54 & 4.91 & 86.32 \\
230 cm (1) & $\boldsymbol{L}$ & 1.01 & 4.98 & 0.43 & 16.99 & 52.22 & 20.40 & 3.96 & 93.58 \\
\hline B. Acacias & $\boldsymbol{W}$ & 2.71 & 11.09 & 1.12 & 33.67 & 9.54 & 36.09 & 5.78 & 85.08 \\
E.D. & $\boldsymbol{B B}$ & 0.97 & 4.57 & 0.42 & 19.66 & 57.76 & 14.84 & 1.79 & 94.04 \\
230 cm (2) & $\boldsymbol{L}$ & 0.91 & 4.35 & 0.60 & 16.52 & 54.06 & 21.20 & 2.36 & 94.14 \\
\hline B. Acacias & $\boldsymbol{W}$ & 1.45 & 3.10 & 0.36 & 17.26 & 53.89 & 17.24 & 6.69 & 95.08 \\
E.D. & $\boldsymbol{B B}$ & 2.10 & 5.02 & 0.35 & 13.23 & 51.02 & 24.81 & 3.47 & 92.53 \\
230 cm (3) & $\boldsymbol{L}$ & 1.75 & 5.07 & 0.48 & 20.30 & 45.19 & 21.45 & 5.76 & 92.71 \\
\hline B.
\end{tabular}

B: Between; E.D.: Ephedra distachya L.; W: Windward; BB: Bush bottom; L: Leeward.

Soil samples had sandy (S), loamy sandy (LS) and sandy loam (SL) textures and clay percentages were very low. Sandy loam (SL) and sandy (S) soils with insufficient organic matter form weakly bound aggregates and are more susceptible to abrasion. On the other hand, generally fine textured soils form strong and resistant aggregates against wind erosion. Any dry and degraded soil is susceptible to wind erosion. As a result, the order of particles resistant to displacement is clay $>$ silt $>$ fine sand. [30].

The amount of lime $\left(\mathrm{CaCO}_{3}\right)$ in the samples varies between $0.0-0.98 \%$ and is classified as low lime. Organic matter is between $0.27-1.06 \%$ and was measured as very little and low $[31,32,33]$. Lime and organic matter protect soils against erosion by increasing aggregation [34] and organic matter is the most effective soil feature for soil erosion [35]. Most soils contain less than $15 \%$ organic matter, and it is less than $2 \%$ in sandy loam soils [36].

The aggregate stability or water-resistant aggregate percentages of sampled soils ranged from 5.56 to 22.65 and were very low. Soils with about $3.5 \%$ organic carbon, or less than 2 percent organic content, can be considered erodible [37]. In general, the sensitivity of soils to erosion decreases with the increase in aggregate stability percentage [38]. The dominant aggregates in agricultural lands are smaller than natural ones, and agricultural soils gain a sensitive structure to erosion by decreasing their aggregate diameter and their stability [39].

The measured $\mathrm{pH}$ values of the samples were between 6.80-8.93 and varied between neutral and strongly alkaline. Soil reaction is effective on the availability of nutrients [40] and the amount of toxic substances formed in the soil. As a result of this, $\mathrm{pH}$ is a soil chemical property that controls plant growth and microorganism activities. Some plants grow only in soils with strong acid reactions, they cannot survive in neutral and alkaline soils; contrary to this, some plants can survive in neutral or alkaline reactions.

The EC. $10^{3}$ values of soil samples were determined to be very salty in all samples. These values are only suitable for the productive growth of certain plant species [41].

According to the results of dry sieving processes, no significant difference was found between samples under control and different protection measures. The percentage of the samples resistant to wind erosion $(>0.84 \mathrm{~mm}$ ) was found to be very low $(0.41-20.81 \%)$, and the percentages of erodible material calculated according to both formulas were found to be quite high (79.19-99.59\% for EF1; 56.67-93.99\% for EF2). The percentage values for the $0.106 \mathrm{~mm}$ diameter material, which increases erosion and erosion by saltation, were found to be quite high (9.54-73.51\%).

According to these results, sampled soils were sensitive to wind erosion according to soil properties, dry sieving results and erodible material percentage values calculated with the help of equations. The lands that are not cultivated throughout Turkey are pasture lands that do not have natural soil cover due to erosion or that most of the very shallow areas have degraded [42]. There was no significant difference between the results of the samples taken from the protected areas and the results of the samples taken from the bare areas.

\subsection{Statistical Analysis Results}


Duncan multiple comparison test results applied to the data for all soil samples used in the research are given in Tables 7, 9 and 11 for case 1, 2 and 3, respectively, with means values and analyses for dry sieve results given in Tables 8,10 and 12 for case 1,2 and 3 , respectively.

Table 7. Statistical results for analyses (Case 1)

\begin{tabular}{cccccccccc}
\hline & Clay & Silt & Sand & Lime & pH & E.C.10 & A. S. & O. M. & EF2 \\
\hline BSA & 2.78 & 19.67 & 77.55 & 0.39 & 8.53 & 162.67 & 15.07 & 0.95 & 66.29 \\
Mean & \pm 1.07 & $\pm 6.49 \mathrm{~A}$ & \pm 7.13 & \pm 0.13 & $\pm 0.19 \mathrm{~A}$ & \pm 34.70 & \pm 1.63 & \pm 0.03 & \pm 4.57 \\
\hline ISA & 2.33 & 18.89 & 78.78 & 0.22 & 8.23 & 90.78 & 13.17 & 0.88 & 66.78 \\
Mean & \pm 0.58 & $\pm 0.38 \mathrm{~A}$ & \pm 0.51 & \pm 0.12 & $\pm 0.29 \mathrm{AB}$ & \pm 3.01 & \pm 2.15 & \pm 0.03 & \pm 2.22 \\
\hline C & 4.67 & 11.00 & 84.33 & 0.54 & 7.86 & 90.76 & 15.09 & 0.65 & 63.61 \\
& \pm 2.31 & $\pm 1.00 \mathrm{~B}$ & \pm 3.21 & \pm 0.39 & $\pm 0.23 \mathrm{~B}$ & \pm 156.96 & \pm 3.49 & \pm 0.33 & \pm 6.30 \\
\hline
\end{tabular}

E.C.10 : Electrical conductivity (dS $\left.\mathrm{cm}^{-1}\right)$; A.S: Aggregate stability (\%); O.M: Organic matter (\%); $E F_{2}$ : Erodible fraction; (\%); BSA: Between Strips of Acacias; ISA: Inside Strips of Acacias; C: Control.

Table 8. Statistical results for dry sieving (Case 1)

\begin{tabular}{|c|c|c|c|c|c|c|c|c|}
\hline & $2 \mathrm{~mm}$ & $1 \mathrm{~mm}$ & $0.84 \mathrm{~mm}$ & $0.42 \mathrm{~mm}$ & $0.106 \mathrm{~mm}$ & $20 \mu$ & $<20 \mu$ & EF1 \\
\hline $\begin{array}{c}\text { BSA } \\
\text { Mean }\end{array}$ & $\begin{array}{c}1.61 \\
\pm 1.21 \mathrm{AB}\end{array}$ & $\begin{array}{c}5.18 \\
\pm 2.83\end{array}$ & $\begin{array}{c}0.53 \\
\pm 0.19\end{array}$ & $\begin{array}{l}16.64 \\
\pm 1.89\end{array}$ & $\begin{array}{l}53.30 \\
\pm 6.58\end{array}$ & $\begin{array}{l}20.43 \\
\pm 2.73\end{array}$ & $\begin{array}{c}2.3 \\
1 \pm 1.33\end{array}$ & $\begin{array}{l}92.68 \\
\pm 4.09\end{array}$ \\
\hline $\begin{array}{c}\text { ISA } \\
\text { Mean }\end{array}$ & $\begin{aligned} & 2.26 \\
& \pm 0.84 \mathrm{~A} \\
&\end{aligned}$ & $\begin{array}{c}5.71 \\
\pm 2.84\end{array}$ & $\begin{array}{c}0.38 \\
\pm 0.15\end{array}$ & $\begin{array}{l}16.69 \\
\pm 3.94\end{array}$ & $\begin{array}{l}51.65 \\
\pm 8.39\end{array}$ & $\begin{array}{l}20.37 \\
\pm 0.97\end{array}$ & $\begin{array}{c}2.94 \\
\pm 0.55\end{array}$ & $\begin{array}{c}91.6 \\
5 \pm 3.79 \\
\end{array}$ \\
\hline C & $\begin{array}{c}0.17 \\
\pm 0.24 \mathrm{~B}\end{array}$ & $\begin{array}{c}1.23 \\
\pm 1.13\end{array}$ & $\begin{array}{c}0.42 \\
\pm 0.20\end{array}$ & $\begin{array}{l}13.44 \\
\pm 4.15\end{array}$ & $\begin{array}{c}66.66 \\
\pm 10.53\end{array}$ & $\begin{array}{l}16.40 \\
\pm 4.85\end{array}$ & $\begin{array}{c}1.68 \\
\pm 1.48\end{array}$ & $\begin{array}{l}98.18 \\
\pm 1.37\end{array}$ \\
\hline
\end{tabular}

For Case 1, according to the analysis results for silt and $\mathrm{pH}$ values and the dry sieving results, $2 \mathrm{~mm}$ sieve values were found to be different from the control values and significant at $\mathrm{p}<0.05$ level. High silt values are negative in terms of erosion because the most susceptible soil particles to erosion are the silt fraction. The increase in $\mathrm{pH}$ values showed higher degree of alkalinity. If there is an increase in $2 \mathrm{~mm}$ diameter particles, and particularly inside the strip increases are in the form of single grains, it is less useful than aggregates for agriculture. Since it has smaller particles in aggregate composition, it is more beneficial for soil and those dependent on soil.

Table 9. Statistical results for analyses (Case 2)

\begin{tabular}{|c|c|c|c|c|c|c|c|c|c|}
\hline & Clay & Silt & Sand & Lime & pH & E.C. $10^{3}$ & A. $S$. & O. M. & EF2 \\
\hline $\begin{array}{c}\text { E.D. } \\
\text { W } \\
\text { Mean }\end{array}$ & $\begin{array}{c}1.89 \\
\pm 0.38 \mathrm{~B} \\
\end{array}$ & $\begin{array}{r}18.44 \\
\pm 3.01 \mathrm{~A} \\
\end{array}$ & $\begin{array}{c}79.67 \\
\pm 3.06 \mathrm{AB} \\
\end{array}$ & $\begin{array}{c}0.19 \\
\pm 0.03 \\
\end{array}$ & $\begin{array}{c}7.44 \\
\pm 0.08 \mathrm{~B} \\
\end{array}$ & $\begin{array}{c}304.11 \\
\pm 58.86 \mathrm{~A} \\
\end{array}$ & $\begin{array}{l}13.49 \\
\pm 2.86 \\
\end{array}$ & $\begin{array}{c}0.87 \\
\pm 0.05 \\
\end{array}$ & $\begin{array}{c}73.94 \\
\pm 4.04 \mathrm{AB} \\
\end{array}$ \\
\hline $\begin{array}{c}\text { E.D. } \\
\text { BB } \\
\text { Mean }\end{array}$ & $\begin{array}{c}1.44 \\
\pm 0.77 \mathrm{~B}\end{array}$ & $\begin{array}{c}20.55 \\
\pm 3.03 \mathrm{~A}\end{array}$ & $\begin{array}{c}78.00 \\
\pm 2.85 \mathrm{~B}\end{array}$ & $\begin{array}{c}0.17 \\
\pm 0.09\end{array}$ & $\begin{array}{c}7.65 \\
\pm 0.16 \mathrm{AB}\end{array}$ & $\begin{array}{c}136.11 \\
\pm 52.55 \mathrm{AB}\end{array}$ & $\begin{array}{l}13.93 \\
\pm 0.06\end{array}$ & $\begin{array}{c}0.85 \\
\pm 0.05\end{array}$ & $\begin{array}{c}78.49 \\
\pm 8.07 \mathrm{~A}\end{array}$ \\
\hline $\begin{array}{c}\text { E.D. } \\
\text { L } \\
\text { Mean }\end{array}$ & $\begin{array}{c}1.89 \\
\pm 0.38 \mathrm{~B}\end{array}$ & $\begin{array}{c}19.11 \\
\pm 3.01 \mathrm{~A} \\
\end{array}$ & $\begin{array}{c}79.00 \\
\pm 3.33 \mathrm{AB} \\
\end{array}$ & $\begin{array}{c}0.21 \\
\pm 0.06\end{array}$ & $\begin{array}{c}7.69 \\
\pm 0.08 \mathrm{AB}\end{array}$ & $\begin{array}{r}118.56 \\
\pm 44.10 \mathrm{AB} \\
\end{array}$ & $\begin{array}{l}14.37 \\
\pm 4.26\end{array}$ & $\begin{array}{c}0.92 \\
\pm 0.09\end{array}$ & $\begin{aligned} & 73.65 \\
\pm & 4.60 \mathrm{AB}\end{aligned}$ \\
\hline C & $\begin{array}{c}4.67 \\
\pm 2.31 \mathrm{~A} \\
\end{array}$ & $\begin{array}{c}11.00 \\
\pm 1.00 \mathrm{~B} \\
\end{array}$ & $\begin{array}{c}84.33 \\
\pm 3.21 \mathrm{~A} \\
\end{array}$ & $\begin{array}{c}0.54 \\
\pm 0.39 \\
\end{array}$ & $\begin{array}{c}7.86 \\
\pm 0.23 \mathrm{~A} \\
\end{array}$ & $\begin{array}{c}90.76 \\
\pm 156.96 \mathrm{~B} \\
\end{array}$ & $\begin{array}{l}15.09 \\
\pm 3.49 \\
\end{array}$ & $\begin{array}{c}0.65 \\
\pm 0.33 \\
\end{array}$ & $\begin{array}{c}63.61 \\
\pm 6.30 \mathrm{~B} \\
\end{array}$ \\
\hline
\end{tabular}

EF $F_{2}$ Erodible fraction; E.D.: Ephedra distachya L.; W: Windward; BB: Bush bottom; L: Leeward; C: Control.

Table 10. Statistical results for dry sieving (Case 2)

\begin{tabular}{|c|c|c|c|c|c|c|c|c|}
\hline & $2 \mathrm{~mm}$ & $1 \mathrm{~mm}$ & $0.84 \mathrm{~mm}$ & $0.42 \mathrm{~mm}$ & $0.106 \mathrm{~mm}$ & $20 \mu$ & $<20 \mu$ & EF1 \\
\hline $\begin{array}{c}\text { E.D. } \\
\text { W } \\
\text { Mean }\end{array}$ & $\begin{array}{c}2.74 \\
\pm 0.68 \mathrm{AB}\end{array}$ & $\begin{array}{r}7.37 \\
\pm 0.65 \mathrm{~A} \\
\end{array}$ & $\begin{array}{c}0.48 \\
\pm 0.15 \\
\end{array}$ & $\begin{array}{r}21.29 \\
\pm 1.11 \mathrm{~A} \\
\end{array}$ & $\begin{array}{c}54.07 \\
\pm 2.67 \mathrm{BC} \\
\end{array}$ & $\begin{array}{l}12.22 \\
\pm 2.54 \\
\end{array}$ & $\begin{array}{r}1.82 \\
\pm 1.71 \\
\end{array}$ & $\begin{array}{c}89.41 \\
\pm 1.11 \mathrm{~B} \\
\end{array}$ \\
\hline $\begin{array}{c}\text { E.D. } \\
\text { BB } \\
\text { Mean }\end{array}$ & $\begin{array}{c}1.15 \\
\pm 0.52 \mathrm{BC} \\
\end{array}$ & $\begin{array}{c}3.61 \\
\pm 1.11 \mathrm{~B} \\
\end{array}$ & $\begin{array}{c}0.28 \\
\pm 0.10 \\
\end{array}$ & $\begin{array}{r}17.35 \\
\pm 3.16 \mathrm{AB} \\
\end{array}$ & $\begin{array}{c}64.27 \\
\pm 4.45 \mathrm{AB} \\
\end{array}$ & $\begin{array}{l}11.46 \\
\pm 2.35 \\
\end{array}$ & $\begin{array}{c}1.89 \\
\pm 1.66 \\
\end{array}$ & $\begin{array}{r}94.96 \\
\pm 1.69 \mathrm{~A} \\
\end{array}$ \\
\hline $\begin{array}{c}\text { E.D. } \\
\text { L } \\
\text { Mean }\end{array}$ & $\begin{array}{r}3.22 \\
\pm 1.70 \mathrm{~A} \\
\end{array}$ & $\begin{array}{r}7.60 \\
\pm 2.21 \mathrm{~A} \\
\end{array}$ & $\begin{array}{c}0.52 \\
\pm 0.13 \\
\end{array}$ & $\begin{array}{r}22.58 \\
\pm 1.66 \mathrm{~A} \\
\end{array}$ & $\begin{array}{c}52.22 \\
\pm 3.28 \mathrm{C} \\
\end{array}$ & $\begin{array}{l}11.97 \\
\pm 1.85 \\
\end{array}$ & $\begin{array}{c}1.88 \\
\pm 1.76 \\
\end{array}$ & $\begin{array}{c}88.66 \\
\pm 3.76 \mathrm{~B}\end{array}$ \\
\hline C & $\begin{array}{c}0.17 \\
\pm .24 \mathrm{C} \\
\end{array}$ & $\begin{array}{c}1.23 \\
\pm 1.13 \mathrm{~B} \\
\end{array}$ & $\begin{array}{c}0.42 \\
\pm 0.20 \\
\end{array}$ & $\begin{array}{c}13.44 \\
\pm 4.15 \mathrm{~B} \\
\end{array}$ & $\begin{array}{c}66.66 \\
\pm 10.53 \mathrm{~A} \\
\end{array}$ & $\begin{array}{l}16.40 \\
\pm 4.85 \\
\end{array}$ & $\begin{array}{c}1.68 \\
\pm 1.48 \\
\end{array}$ & $\begin{array}{r}98.18 \\
\pm 1.37 \mathrm{~A} \\
\end{array}$ \\
\hline
\end{tabular}


For case 2, according to the analysis results for clay, silt, sand, $\mathrm{pH}, \mathrm{EC} .10^{3}$ and EF2 values; and the dry sieving results, 2, $1,0.42,0.106 \mathrm{~mm}$ sieve values and percentages of EF1 were found to be different from the control values and significant at $p$ $<0.05$ level. The difference in clay values is toward a decrease and is a negative situation. The difference in silt values is toward an increase and is a negative situation in terms of susceptibility to erosion. The difference in sand values is in a decrease and is a positive situation. The decrease in $\mathrm{pH}$ values was from higher alkalinity to lower values and this was considered positive. The increase in EC. $10^{3}$ values indicate an increase in salinity and a negative situation. The increase in EF2 values is an undesirable negative situation. If the increase in 2- and 1-mm sieve values is in the form of individual grains, it is more useless for agriculture than aggregates. The increase in $0.42 \mathrm{~mm}$ sieve value was evaluated as beneficial in terms of the increment in particles larger than $0.106 \mathrm{~mm}$ critical diameter causing saltation. The decrease in the $0.106 \mathrm{~mm}$ sieve value was evaluated as a positive situation in terms of decreasing saltation during wind erosion and a negative situation in terms of particles suspended in the air. The decrease in EF1 values is a positive difference.

Table 11. Statistical results for analyses (Case 3)

\begin{tabular}{|c|c|c|c|c|c|c|c|c|c|}
\hline & Clay & Silt & Sand & Lime & pH & E.C. $10^{3}$ & A. $S$. & O. M. & EF2 \\
\hline $\begin{array}{c}\text { IA E.D. } \\
\text { Mean }\end{array}$ & $\begin{array}{c}2.33 \\
\pm 0.67 \\
\end{array}$ & $\begin{array}{l}22.44 \\
\pm 2.78 \\
\end{array}$ & $\begin{array}{c}75.22 \\
\pm 3.01 \mathrm{~B} \\
\end{array}$ & $\begin{array}{c}0.18 \\
\pm 0.02 \\
\end{array}$ & $\begin{array}{c}7.87 \\
\pm 0.04 \\
\end{array}$ & $\begin{array}{c}203.22 \\
\pm 121.40 \\
\end{array}$ & $\begin{array}{l}12.52 \\
\pm 4.51 \\
\end{array}$ & $\begin{array}{c}0.86 \\
\pm 0.10 \\
\end{array}$ & $\begin{array}{l}70.01 \\
\pm 3.69 \\
\end{array}$ \\
\hline C & $\begin{array}{c}4.67 \\
\pm 2.31\end{array}$ & $\begin{array}{l}11.00 \\
\pm 1.00\end{array}$ & $\begin{array}{c}84.33 \\
\pm 3.21 \mathrm{~A}\end{array}$ & $\begin{array}{c}0.54 \\
\pm 0.39\end{array}$ & $\begin{array}{c}7.86 \\
\pm 0.23\end{array}$ & $\begin{array}{c}90.76 \\
\pm 156.96\end{array}$ & $\begin{array}{l}15.09 \\
\pm 3.49\end{array}$ & $\begin{array}{c}0.65 \\
\pm 0.33\end{array}$ & $\begin{array}{l}63.61 \\
\pm 6.30\end{array}$ \\
\hline
\end{tabular}

Table 12. Statistical results for dry sieving (Case 3)

\begin{tabular}{cccccccccc}
\hline & $\mathbf{2} \mathbf{~ m m}$ & $\mathbf{1} \mathbf{~ m m}$ & $\mathbf{0 . 8 4} \mathbf{~ m m}$ & $\mathbf{0 . 4 2} \mathbf{~ m m}$ & $\mathbf{0 . 1 0 6} \mathbf{~ m m}$ & $\mathbf{2 0} \boldsymbol{\mu}$ & $<\mathbf{2 0} \boldsymbol{\mu}$ & EF1 \\
\hline IA E.D. & 2.26 & 6.20 & 0.50 & 19.46 & 45.02 & 22.41 & 4.17 & 91.05 \\
Mean & $\pm 1.07 \mathrm{~A}$ & $\pm 1.27 \mathrm{~A}$ & \pm 0.15 & \pm 3.06 & $\pm 8.27 \mathrm{~B}$ & \pm 2.37 & \pm 0.86 & $\pm 2.38 \mathrm{~B}$ \\
\hline \multirow{2}{*}{$\mathbf{C}$} & 0.17 & 1.23 & 0.42 & 13.44 & 66.66 & 16.40 & 1.68 & 98.18 \\
& $\pm 0.24 \mathrm{~B}$ & $\pm 1.13 \mathrm{~B}$ & \pm 0.20 & \pm 4.15 & $\pm 10.53 \mathrm{~A}$ & \pm 4.85 & \pm 1.48 & $\pm 1.37 \mathrm{~A}$ \\
\hline
\end{tabular}

For case 3, according to the analysis results sand and dry sieving results, sieve values for 2, 1 and $0.106 \mathrm{~mm}$ and EF1 percentages were found to be different from the control values and significant at the $\mathrm{p}<0.05$ level. The difference in sand values is a decrease and can be accepted as a positive situation, but the decrease in the clay value and the increase in the silt value are negative in terms of erosion. The increases in 2- and 1-mm sieve values are less favourable in terms of individual grains than aggregates. The decrease in the $0.106 \mathrm{~mm}$ sieve value was evaluated as a positive situation in terms of decreasing saltation during wind erosion and a negative situation in terms of particles suspended in the air.

\section{Conclusions and Recommendations}

Accelerated erosion because of natural disasters and anthropogenic effects is a result of vegetation deficiency and partly mistaken cultural processes [43]. Vegetation is one of the important factors controlling erosion. The vegetation on the soil surface keeps the soil particles together with developed root systems, reduces the wind speed close to the ground level on the soil and prevents the transport of soil particles. However, natural plant communities in semi-arid and arid climates cannot fully protect the soil surface from the erosive forces of the wind. Semiarid ecosystems, including grasslands, scrub and scrub lands, savannah, woodlands, and forests, are highly susceptible to wind erosion, especially when degraded by human influences [44].
Loss or insufficient protective vegetation is generally evaluated together with changes in physicochemical and biological soil properties such as soil structure, plant nutrient availability, organic matter content and microbial activities. About wind erosion control, it is critical to address not only vegetation, but also these biological and physicochemical soil characteristics [45].

The results of the study indicate insufficient $(<75 \%)$ existing vegetation in protected areas and revealed that there is very little vegetation (10-40\%) in unprotected areas [14] and the negative effects of wind erosion will continue if the cover percentage is not increased. Generally, statistical results were unfavourable and confirmed this view.

The distance between the two rows of acacia strips is 25 meters and it is suitable for the literature, but the planting of two rows was performed incorrectly. Inside facing rows should be short and provide ground level cover [30]. Since acacia trees are the same height and their lower parts remain open, their protection power remained low. On the other hand, shorter edges were left open while creating acacia strips. This indicates that the protection process started incompletely. Planning according to the prevailing wind is important, but not sufficient. Wind shear can come from all directions; therefore, all sides of the land to be protected should be surrounded by windbreakers so as not to interfere with the intended use. 


\section{References}

[1] Karlen, D.L., Andrews, S.S. Wienhold, B.J., \& Doran, J. W. (2003). Soil quality: Humankind's foundation for survival. J. Soil Water Conservation, 58, 171-179.

[2] Altın, M., Gökkuş, A., \& Koç, A., (2011). Meadow and Rangeland Management (In Turkish). $2^{\text {nd }}$ Volume (Basic principles). TUGEM Publications, pp. 84-88, Ankara, Turkey.

[3] Ahmad, K., Ashraf, M., Khan, Z. I., \& Valeem R. E. (2008). Evaluation of Macro-Mineral Concentrations of Forages in Relation to Ruminants Requirements: A Case Study in Soone Valley, Punjab. Pakistan. Pak. Journal of Bot., 40(1), 295299.

[4] Ghazanfar, S., Latif, A., Mirza, I. H., \& Nadeem, M. A. (2011). Macro-Minerals Concentrations of Major Fodder Tree Leaves and Shrubs of District Chakwal, Pakistan. Pak. Journal Nutr., 10(5), 480-484.

[5] Louhaichi, M., Salkini, A.K., \& Petersen, S. L. (2009). Effect of small ruminant grazing on the plant community characteristics of semiarid Mediterranean ecosystems. Int. J. Agric. Biol., 11, 681-689.

[6] Zobeck, T.M., Popham, T.W., Skidmore, E. L., Lamb, J.A., Merril, S.D., Lindstrom, M.J., Mokma, D.L., \& Yoder, R. E. (2003). Aggregate-mean diameter and wind-erodible soil predictions using dry aggregate-size distributions. Soil Sci. Soc. Am. J. 67, 425-436.

[7] Goossens, D., \& Riksen, M. J. P. M. (2007). The role of wind and splash erosion in inland drift-sand areas in the Netherlands. Geomorphology, 88(1- 2), 179-192.

[8] Acar, R., \& Dursun, S. (2010). Vegetative methods to prevent wind erosion in Central Anatolia Region. Int. J. of Sustainable Water \& Environmental Systems, 1(1), 25-28.

[9] Karaoğlu, M. (2018a). Wind erosion studies in Iğdır-Aralik. Journal of Agriculture, 1(2), 25-38, Iğdır, Turkey.

[10] Özdoğan, N. (1976). Wind erosion and major measures to be taken in wind erosion areas (In Turkish). General Directorate of Soilwater, General publication No: 306. Ankara, Turkey.

[11]Li, J., Okin, G. S., \& Epstein, H. E. (2009). Effects of enhanced wind erosion on surface soil texture and characteristics of windblown sediments. Journal of Geophysic. Res., 114, 1-8.

[12] Fryrear, D. W., Krammes, C. A., Williamson, D. L., \& Zobeck, T. M., (1994). Computing the wind erosion fraction of soils. Soil Water Conservation. 49, 183-188.,

[13]Zobeck, T. M., Popham, T. W., Skidmore, E. L., Lamb, J. A. Merril, S. D., Lindstrom, M. J., Mokma, D. L., \& Yoder, R. E. (2003) Aggregate-mean diameter and wind-erodible soil predictions using dry aggregate-size distributions. Soil Sci. Soc. Am. J. 67, 425-436.

[14] Karaoğlu, M., Şimşek, U., Tohumcu, F., \& Erdel, E. (2017). Determining surface soil properties of wind erosion area of Igdir-Aralik and estimating the soil loss. Fresenius Environmental Bulletin, 26(5), 3170-3175.

[15] Chepil, W. S. (1962). A compact rotary sieve and the importance of dry sieving in physical soil analysis. Soil Sci. Soc. Am. Proc. 26, 4-6.

[16] Colazo, J. C., \& Buschiazzo D. E. (2010). Soil dry aggregate stability and wind erodible fraction in a semiarid environment of Argentina. Geoderma 159: 228-236.,

[17] Garrett, H. E., \& Buck, L. E. (1997). Agroforestry practice and policy in the United States of America. For Ecology
Management, 91, 5-15.

[18] Gee, G. W., \& Bauder, J. W. (1986). Particle-size analysis. p. 383-411. In A. Klute (ed.) Methods of soil analysis. Part 1. 2nd ed. Agron. Monogr. 9. ASA and SSSA, Madison, WI.

[19] Mclean, E. O. (1982). Soil pH and lime requirement. In Page, A. L., R. H. Miller and D. R. Keeney (eds.) Methods of soil analysis. Part 2 - Chemical and microbiological proper-ties. ( $2^{\text {nd }}$ Ed.). Agronomy, 9, 199-223.

[20] Nelson, R. E. (1982). Carbonate and gypsum. P. 181-197. In A.L. Page et al. (ed). Methods of soil analysis. Part 2. 2nd ed. Agron. Monogr. 9. ASA and SSSA, Madison, WI.

[21] Walkley A, \& Black LA, (1934). An examination of the degtjareff method for determining soil organic matter, and a proposed modification of the chromic acid titration method. Soil Science, 37 (1): 29-38. doi: https://doi.org/10.1097/00010694-193401000-00003.

[22] Demiralay, İ. (1993). Soil physical analysis (In Turkish). Erzurum Atatürk University Publications No: 143, Erzurum, Turkey.

[23] Chepil, W. S. (1953). Factors that influence clod structure and erodibility of soil by wind: I. Soil texture. Soil Sci. 75, 473-483.

[24] Hagen, L. J., van Pelt, S., \& Sharratt, B. (2010). Estimating the saltation and suspension components from field wind erosion. Aeolian Research, 1, 147-153.

[25] Chepil, W. S. (1942). Measurement of wind erosiveness by dry sieving procedure. Sci. Agric. 23, 154-160.

[26] Lyles, L., \& Woodruff, N. P. (1960). Surface soil cloddiness in relation to soil density at time of tillage. Soil Sci., 91, 178182.

[27] Chepil, W. S. (1955). Factors that influence clod structure and erodibility of soil by wind: V. Organic matter at various stages of decomposition. Soil Sci., 80, 413-421.

[28] Chepil, W. S. (1954). Factors that influence clod structure and erodibility of soil by wind: III. Calcium carbonate and decomposed organic matter. Soil Sci., 77, 473-480.

[29] Açıkgöz, N. (1993). Research and experiment methods in agriculture (In Turkish). E.U.A.F. Pub., No: 478, $3^{\text {rd }}$ Edit., İzmir, Turkey.

[30] Blanco, H., \& Lal, R. (2008). Principles of Soil Conservation and Management. Springer Science + Business Media B.V. ISBN: 978-1-4020-8708-0.

[31] Ülgen, N., \& Yurtsever, N. (1974). Turkey Fertilizer and Manure Guide (In Turkish). Soil and Fertilizer Research Institute Technical Publications Series No: 28, Kemal Printery, Ankara, Turkey.

[32] Aydın, A., \& Sezen, Y. (1995). Soil chemistry laboratory book (In Turkish). E.A.U. Agricultural Faculty Course Publications No: 174, 146 pages, Offset plant, Erzurum, Turkey.

[33] Güçdemir, İ. H. (2008). Sampling for Soil, Water and Plant Analysis, Fertilizers, Fertilization Based on Soil Analysis. Ministry of Agriculture and Rural Affair, General Pub. No: 68, Farmer Pub. No: 3, Ankara.

[34] Taysun, A., Saatçı, F., \& Uysal, H. (1984). A preliminary study on the effect of PVA (Polyvinylalcohol) application on soils on aggregation. E. U. A. F. Journal, 21(3), 27-33.

[35] Wischmeier, W. D., \& Mannering, J. V. (1969). Relation of soil properties and erodibility. Soil Sci. Soc. Amer. Proc., 33, 131-137.

[36] Morgan, R. P. C. (2005). Soil Erosion \& Conservation. $3^{\text {rd }}$ Ed. Blackwell publishing, 108 Cowley Road, Oxford OX4 1JF, UK. 
[37]Evans, R. (1980). Mechanics of water erosion and their spatial and temporal controls: an empirical viewpoint. In Kirkby, M.J. and Morgan, R.P.C. (eds), Soil erosion. Wiley, Chichester, 109-128.

[38] Tate, R. L. (2000). Soil Microbiology. 2nd Edition. 536 Pages. John Wiley \& Sons, ISBN: 978-0-471-31791, New York.

[39] Taysun, A. (1986). Research on the effects of stones, plant residues and polyvinyl alcohol (PVA) on soil properties and erosion with the help of artificial sprinklers in rendzina agricultural soils in Gediz basin (Thesis of Assoc. Prof. Dr. In Turkish). E.U. Agr. Fac. Pub. No: 474.

[40] Sönmez, S. (2013). Basic compounds of plant nutrition: Water, soil, nutrients (In Turkish). Hasad Pub., 176. s, ISBNp: 9789758377886.

[41] Aydemir, O. (1992). Plant nutrition and soil fertility (In Turkish). Erzurum Atatürk University Publications. No: 734,
Erzurum, Turkey.

[42] Çelik, A., \& Akça, E. (2021). Description of the quartercentury effect of conversion from rainfed Farming to irrigated farming on a micromorphological scale. European Journal of Science and Technology, 21, 207-215.

[43] Karaoğlu, M. (2018b). The importance of vegetation in wind erosion. Journal of Agriculture, ISSN: 2636-8757, 1(2), 4960, Igdir, Turkey.

[44]Li, J., Okin, G.S. Alvarez, L., \& Epstein, H. (2007). Quantitative effects of vegetation cover on wind erosion and soil nutrient loss in a desert grass- land of southern New Mexico. Biogeochemistry, 85, 317-322.

[45] Van Pelt, R. S., \& Zobeck, T. M. (2004). Effects of polyacrylamide, cover crops, and crop residue management on wind erosion, ISCO, 13th International Soil Conservation Organisation Conference, Brisbane. 